\title{
IONIC EQUILIBRIA IN PROTIC AND IN DIPOLAR APROTIC SOLVENTS
}

\author{
A. J. PARKER \\ Research School of Chemistry, Australian National University, \\ Canberra, A.C.T. 2600, Australia
}

\begin{abstract}
'Chemical interactions' as well as 'physical interactions' play an important part in solvation. Some equilibria are reversed on transfer from protic to dipolar aprotic solvents and this behaviour can be interpreted and predicted through the solvent activity coefficients of the appropriate solutes. Some extrathermodynamic assumptions for the evaluation of single ion solvent activity coefficients are discussed. Trifluoroethanol seems to solvate anions more than does ethanol but solvates cations less than does ethanol.

The Principle of Hard and Soft Acids and Bases is valid in dipolar aprotic solvents as well as in water. Solvation of transition states and of ion pairs is compared. Transition states are in equilibrium with their environment. Enthalpies of transfer parallel free energies of transfer, when a series of salts of the one cation are considered.
\end{abstract}

Our interest in ionic equilibria in dipolar aprotic solvents began fifteen years ago, when we noted the enormous increase in rate of many important organic reactions on transfer from alcohols to solvents like dimethylformamide and dimethylsulphoxide ${ }^{1}$. Our work since then has always been from the point of view of the physical organic chemist ${ }^{2}$, rather than of the electrochemist, analyst, or physical chemist, so that the work that I will describe contains all the strengths and weaknesses that this approach implies. It must be realised that solvent transfer frequently creates changes of one million or more in a rate or equilibrium constant, so that some uncertainties and approximations in available data can be tolerated.

Rates of slow reactions, like the $\mathrm{S}_{\mathrm{N}} 2$ exchange between chloride ion and methyl iodide in solution, are best interpreted in terms of the absolute rate

$$
\begin{aligned}
& \text { Rate } \quad \mathrm{CH}_{3} \mathrm{I}+\mathrm{Cl} \stackrel{\mathrm{K \ddagger}}{\rightleftharpoons} \mathrm{Cl}-\overbrace{\mathrm{H}}^{\mathrm{C}} \stackrel{\mathrm{H}}{\mathrm{H}} \mathrm{I}^{\ddagger-} \rightarrow \mathrm{ClCH}_{3}+\mathrm{I}^{-} \\
& k^{\mathrm{s}} / k^{\mathrm{o}}=\frac{{ }^{\mathrm{o}} \gamma_{\mathrm{CH}_{3} \mathrm{I}}^{\mathrm{s}} \times{ }^{\mathrm{o}} \gamma_{\mathrm{Cl}^{-}}^{\mathrm{s}}}{{ }_{0} \gamma_{+}^{\mathrm{s}}-} \\
& \text { Equilibrium } \quad \mathrm{AgI}+\mathrm{Cl}^{-} \stackrel{K}{\rightleftharpoons} \mathrm{ClAgI}^{-}
\end{aligned}
$$


A. J. PARKER

$$
K^{\mathrm{s}} / K^{\mathrm{o}}=\frac{{ }_{\gamma_{\mathrm{AgI}}^{\mathrm{s}}}^{\mathrm{s}} \times{ }^{{ }^{o}} \gamma_{\mathrm{Cl}^{\mathrm{s}}-}^{\mathrm{s}}}{{ }^{0} \gamma_{\mathrm{ClAgI}}^{\mathrm{s}}{ }^{-}}
$$

or transition state theory. There is an equilibrium between reactants and a transition state and also between the transition state and its environment. This last point is important, because it means that a transition state is solvated just like any other related solute. Thus it is reasonable to use an associative equilibrium, such as that between dissolved silver iodide and chloride ion, as a model for an associative rate process and to compare the effect of solvent transfer on the 'real' equilibrium constant and on the 'kinetic' equilibrium constant. We can then obtain information about the solvation of the transition state. This allows us to deduce something about the reaction mechanism ${ }^{3}$, because we can compare the solvent activity coefficient of the transition state with the solvent activity coefficients of various model solutes and see which model is closest to the transition state. In this example, ${ }^{\circ} \gamma_{i}^{s}$ are solvent activity coefficients, reflecting the change in the standard chemical potential of a solute $i$, which is hypothetically ideal in a unimolar solution, on transfer from a reference solvent $\mathrm{O}$ to another solvent $\mathrm{S}$.

Solvent activity coefficients, as well as equilibrium constants in different solvents ${ }^{4}$, are often conveniently obtained by electrochemical measurements, so that there is an important link between reaction mechanism, kinetics, equilibria and electrochemistry. This is one reason for my interest in this symposium and I would emphasize the obvious point, that the measurements being made by electrochemists do have important implications outside these specific fields.

It is important that we appreciate the role of 'chemical interactions' in solvation, especially in solvents of much the same dielectric constant. By 'chemical interactions' we mean interactions like 'hydrogen bonding', Lewis acid-base, solvent structure, hard-soft interactions, and so on; they contrast with 'physical interactions' which are associated with coulombic forces, the solvent dielectric constant, and solute size.

Some effects of chemical interactions on some silver-chloride ionic equilibria are illustrated in Table 1. Methanol, acetonitrile, nitromethane, and hexamethylphosphoramide are solvents with much the same dielectric constant. However, the dielectric constant tells us very little about likely solvent effects on these associative equilibria, because the solubility of silver chloride is very different in nitromethane, although similar in the other solvents. The stability of the silver dichloride anion is very different in methanol versus hexamethylphosphoramide, and different again in acetonitrile and nitromethane. Clearly we cannot only consider electrostatics, but must also consider the different chemical interactions of the solutes with each solvent. The differences are predictable, but it is necessary to consider hydrogen bonding to chloride ion, acid-base interactions of silver with the solvents, and perhaps even the hardness and softness of solutes and solvents, if we are to explain the variations in equilibrium constants shown in Table 1.

This is a typical example of the large changes in equilibrium constants, when systems are transferred from protic to dipolar aprotic solvents. It is even more instructive to look at some very simple equilibria such as acid- 
Table 1. Chemical interactions in 'isodielectric' solvents at $25^{\circ}$

$$
\mathrm{Ag}^{+}+2 \mathrm{Cl}^{-} \stackrel{K_{\mathrm{SO}}}{=} \mathrm{AgCl} \downarrow+\mathrm{Cl}^{-} \stackrel{K_{\mathrm{S} 2}}{=} \mathrm{AgCl}_{2}^{-}
$$

\begin{tabular}{|c|c|c|c|c|}
\hline Solvent & $\mathrm{CH}_{3} \stackrel{\partial-}{\mathrm{O}}-\stackrel{\partial+}{\mathrm{H}}$ & $\mathrm{CH}_{3} \stackrel{\partial+}{\mathrm{C}} \equiv \stackrel{\partial-}{\mathrm{N}}$ & $\mathrm{H}_{3} \stackrel{+}{\mathrm{N}}$ & $\left(\mathrm{Me}_{2} \stackrel{\partial+}{\mathrm{N}}\right)_{3} \mathrm{P}=\stackrel{\partial-}{\mathrm{O}}$ \\
\hline$\varepsilon$ & 32.6 & 37.5 & 38.6 & 29.6 \\
\hline $\begin{array}{l}\log K_{\mathrm{So}} \\
\log K_{\mathrm{S} 2}\end{array}$ & $\begin{array}{r}13.1^{\mathrm{a}} \\
-5.2^{\mathrm{a}}\end{array}$ & $\begin{array}{r}12.9^{\mathrm{a}} \\
+0.2^{\mathrm{a}}\end{array}$ & $\begin{array}{r}19.8^{\mathrm{b}} \\
+0.3^{\mathrm{b}}\end{array}$ & $\begin{array}{r}11.9^{\mathrm{a}} \\
+4.2^{\mathrm{a}}\end{array}$ \\
\hline
\end{tabular}

a Alexander, E. C. F. Ko, Y. C. Mac and A. J. Parker, J. Amer. Chem. Soc. 89, 3703 (1967).

Reference 4.

base, ion pairing, and solubility. The equilibria all involve chloride ion and would be familiar to all freshmen chemists. They would tell you, correctly, that all these equilibria lay to the right in water. Equilibrium constants for acid-base equilibria, for ion pairing, for solubility and for instability in aqueous solutions are still sometimes discussed by teachers, and in text books, in terms of the intrinsic properties of the chloride ion and of the other species. However the situation in water may give a very distorted picture and the gas phase equilibria may lie in quite the opposite direction. This is suggested by the fact that of the equilibria shown, all lie to the left in some dipolar aprotic solvents, whereas, as noted, all lie to the right in water and methanol at $25^{\circ}$.

Reversal of Chloride Ion Equilibria on Solvent Transfer

$\leftarrow$ Dipolar Aprotic (e.g. $\mathrm{CH}_{3} \mathrm{CN}$ )

Protic $\rightarrow$

(e.g. $\mathrm{H}_{2} \mathrm{O}$ )

$\mathrm{AuCl}_{2}^{-}+2 \mathrm{SCN}^{-} \rightleftharpoons \mathrm{Au}(\mathrm{SCN})_{2}^{-}+2 \mathrm{Cl}^{-} \quad$ Lewis Acid-Base

$\mathrm{Me}_{4} \mathrm{~N} \cdot \mathrm{Cl}+\mathrm{Pic}^{-} \rightleftharpoons \mathrm{Me}_{4} \mathrm{~N}$.Pic $+\mathrm{Cl}^{-} \quad$ Ion Pair

$\mathrm{KCl} \downarrow+\mathrm{ClO}_{4}^{-} \rightleftharpoons \mathrm{KClO}_{4} \downarrow+\mathrm{Cl}^{-} \quad$ Solubility

$\mathrm{AgCl}_{2}^{-} \quad \rightleftharpoons \mathrm{AgCl} \downarrow+\mathrm{Cl}^{-} \quad$ Complexing

It is necessary to understand ion-solvent interactions before we can begin to understand such solvent effects on equilibria. The topic will be discussed by Professor Strehlow but I want to consider qualitatively, five types of interaction ${ }^{3}$. These are coulombic ion-dipole interactions, hydrogen bonding interactions, dispersion forces, solvent structure, and Lewis acidbase interactions. Some people who follow Pearson's principle of Hard and Soft Acids and Bases may prefer to put these all under the one heading of hard or soft interactions ${ }^{5}$, but $I$ think that this would obscure a great deal of chemistry. 


\section{A. J. PARKER}

A qualitative picture of the influence of these interactions on the standard chemical potential of seven representative anions, is shown in Figure $1^{3}$. Large polarizable anions are to the left, small anions are to the right. The picture shows four curves. All anions are unsolvated in vacuo, so at the top, we represent no interactions by a line of zero slope with all anions having solvent activity coefficients of unity. The sloping straight line is a Born curve, representing the greater coulombic solvation of smaller anions in a medium

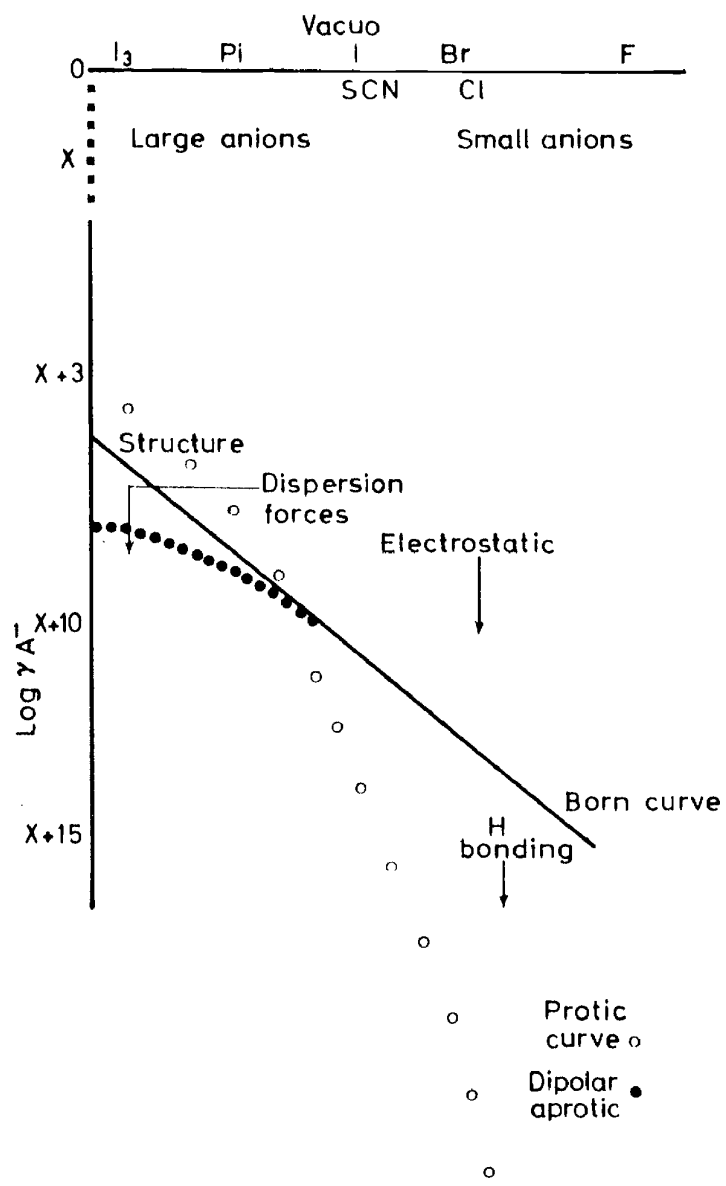

Figure 1. A qualitative representation (as $\log { }^{0} \gamma_{Y^{-}}^{\mathrm{s}}$ ) of the effect of electrostatic, hydrogen bonding, mutual polarizability and structural interactions, between anion and solvent, on the standard chemical potential of anions on transfer from vacuum to a protic and a dipolar aprotic solvent each of dielectric constant 35 cf. Reference 3

of dielectric constant 35. The curve of filled circles is for a hypothetical dipolar aprotic solvent like dimethylformamide, but of a dielectric constant 35 , and the dotted curve of open circles is for a hypothetical protic solvent, like water, but also of dielectric constant 35 . The dipolar aprotic curve has a 
downwards deviation from the Born curve showing greater solvation than expected for very polarizable anions. This represents dispersion force interactions of large polarizable anions with polarizable dipolar aprotic solvents. The protic solvent curve shows a strong downwards deviation which is greatest for small anions which are the strongest hydrogen bond acceptors, and represents increased solvation due to hydrogen-bond interactions with protic solvents. There is also an upwards deviation of the protic curve from the Born line, which is greatest for the largest anions and represents decreased solvation because of unfavourable structural interactions with a highly structured protic solvent like water. The effects of dispersion forces are very small in our model protic solvent like water, so no deviations are shown for the protic solvent, likewise the effects of hydrogen bond donation to the anion are negligible in our model dipolar aprotic solvent, like dimethylformamide, so no deviations are shown for small anions in the aprotic solvent.

Some attempt has been made to show the relative importance of the interactions to scale in the picture. The interactions were gauged in a variety of ways. I agree with Alfenaar and de Ligny ${ }^{6}$ that the effects of solvent structure and dispersion forces are reflected in the solvent activity coefficients of the inert gases. Ferrocene is also a good indicator of these interactions ${ }^{6,7}$. Some solvent activity coefficients, for transfer from water, are in Table 2 for 'inert' solutes. Their different sizes are shown as reciprocals of their crystal radius ${ }^{6}$, so that the smallest solutes are represented by the largest numbers in reciprocal ångströms.

Table 2. Structure and dispersion forces

Solvent activity coefficients of inert non-electolytes Transfer from water at $25^{\circ}$

\begin{tabular}{lcccccccccc}
\hline \multicolumn{2}{c}{ Size } & & \multicolumn{8}{c}{$\log ^{\mathrm{w}_{\gamma^{\mathrm{s}}}}$} \\
\multicolumn{1}{c}{ Solute } & $1 / \mathrm{r}^{\mathrm{b}}$ & $\mathrm{S}: \mathrm{H}_{2} \mathrm{O}$ & $\mathrm{MeOH}$ & $\mathrm{Me}_{2} \mathrm{CO}$ & $\mathrm{CH}_{3} \mathrm{CN}$ & $\mathrm{CH}_{3} \mathrm{NO}_{2}$ & $n-\mathrm{C}_{6} \mathrm{H}_{14}$ & DMSO & $\mathrm{DMF}$ \\
\hline Neon & 0.625 & $0.0^{\mathrm{a}}$ & $-0.6^{\mathrm{a}}$ & $-0.6^{\mathrm{c}}$ & - & $-0.4^{\mathrm{e}}$ & $-0.8^{\mathrm{c}}$ & $-0.1^{\mathrm{a}}$ & - \\
Xenon & 0.450 & $0.0^{\mathrm{a}}$ & $-1.7^{\mathrm{a}}$ & $-1.7^{\mathrm{a}}$ & $-1.7^{\mathrm{e}}$ & $-1.1^{\mathrm{e}}$ & $-1.6^{\mathrm{c}}$ & $-0.7^{\mathrm{a}}$ & - \\
Ferrocene & 0.263 & $0.0^{\mathrm{a}}$ & $-3.6^{\mathrm{a}}$ & $-4.0^{\mathrm{d}}$ & $-3.6^{\mathrm{d}}$ & - & - & - & $-4.1^{\mathrm{d}}$ \\
& & & $\mathrm{Li}^{+}=1.47 \AA^{-1} ;$ & $\mathrm{Cs}^{+}=0.59 \AA^{-1}$ & & & \\
\hline
\end{tabular}

Reference 3

b Reference 6

H. Stephen and T. Stephen, Solubilities of Inorganic and Organic Compounds, Pergamon Press, London (1963).

d Reference 7 .

- Fan-tih Chiang, Ph.D. Thesis, University of Minnesota (1967).

Neon, xenon and ferrocene are all more solvated by methanol, acetone, acetonitrile, hexane, and dimethylsulphoxide than by water, but if we exclude water, these five rather different solvents, solvate an inert solute to much the same extent.

It is only when we transfer inert solutes from water that we have significant changes in solvent activity coefficients. Xenon for example is 


\section{A. J. PARKER}

similarly solvated by methanol, acetone, acetonitrile and hexane. The combination of structural and dispersion force interactions apparently is fairly constant through the series of non-aqueous solvents shown. Water behaves in a special way. As the size and polarizability of the solute increase, the solute becomes increasingly less solvated by water, relative to other solvents, so that calculation of single ion solvent activity coefficients through a modified Born equation ${ }^{8}$, or by assumptions about the coulombic interactions of large cations, like rubidium ${ }^{9}$, are unlikely to be realistic, if water is the reference solvent. Some allowance for structure and dispersion forces must be made when considering transfer from water to non-aqueous solvents ${ }^{6}$. The non-electrostatic contribution to solvation of the very small lithium cation $\left(1 / r=1.47 \AA^{-1}\right)$ is likely to be much less than that for the much larger cesium cation $\left(1 / \mathrm{r}=0.59 \AA^{-1}\right)$ in water, but it may be similar for these cations in other solvents like sulpholane or methanol ${ }^{8}$. The behaviour of the inert gases on solvent transfer suggest that calculations of single ion solvent activity coefficients, which are based on a modified Born equation $^{8}$, are more likely to be successful if some solvent other than water is chosen as reference solvent.

We showed very large deviations from the Born curve for hydrogen-bond interactions of anions with protic solvents. Some justification for regarding this as a major interaction in protic solvents is shown in Table 3 for transfer

Table 3. Solvent activity coefficients of anions $\mathrm{s}^{\mathrm{a}}$

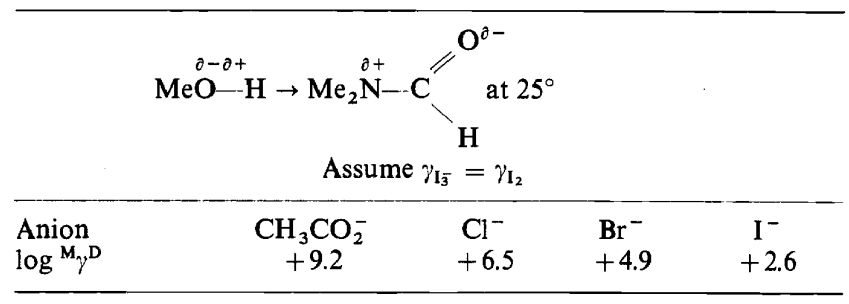

a Reference 3.

of four anions from methanol to dimethylformamide. The anions have decreasing tendencies to accept hydrogen bonds from left to right, acetate is a strong hydrogen bond acceptor, iodide ion is a weak hydrogen bond acceptor. Methanol is a hydrogen bond donor, dimethylformamide is not, so that anions like acetate, are much less solvated by dimethylformamide than by methanol, that is they have solvent activity coefficients, for transfer from methanol to dimethylformamide, which are much greater than unity, i.e. positive logarithmic values. The solvent activity coefficients decrease as the anions become less powerful hydrogen bond acceptors. Most of the decrease shown from acetate to iodide ion in Table 3, is thought to be a reflection of decreasing hydrogen bond interactions with methanol, as the anion becomes larger. 
Cations sometimes have strong acid-base interactions with solvents. Some examples of solvent activity coefficients for cations are shown in Table 4 for transfer from water to acetonitrile. The very small solvent activity coefficients of silver I and copper I cations, which are shown as negative $\log$ values, are thought to be an indication of stronger specific interactions of the Lewis acids, silver and cuprous cation, with the Lewis base acetonitrile, than with water. The closed shell cations, like potassium, thallium, and copper II, are similarly solvated by water and acetonitrile, i.e. they have solvent activity coefficients relatively close to unity.

Table 4. Solvent activity coefficients of cations

\begin{tabular}{|c|c|c|c|c|c|}
\hline & & $\underset{\text { Ass }}{\mathrm{HO}-\mathrm{H}}$ & $\begin{array}{l}\mathbf{C +} \stackrel{0}{=} \mathbf{N} \text { at } \\
=\gamma_{\mathrm{I}_{2}}\end{array}$ & & \\
\hline $\begin{array}{l}\text { Cation } \\
\log \mathrm{w}_{\gamma} \mathrm{A}\end{array}$ & $\begin{array}{l}\mathrm{K}^{+} \\
+0.7^{\mathrm{a}}\end{array}$ & $\begin{array}{l}\mathrm{Tl}^{+} \\
-0.8^{\mathrm{a}, \mathrm{b}}\end{array}$ & $\begin{array}{l}\mathrm{Cu}^{2+} \\
+1.0^{\mathrm{c}}\end{array}$ & $\begin{array}{l}\mathrm{Cu}^{+} \\
-12.5^{\mathrm{c}}\end{array}$ & $\begin{array}{l}\mathrm{Ag}^{+} \\
-5.5^{\mathrm{a}}\end{array}$ \\
\hline
\end{tabular}

"Reference 3 .

${ }^{b}$ Reference 8 .

' J. H. Sharp and A. J. Parker, unpublished work, cf. S. E. Manahan and R. T. Iwamoto, Inorg. Chem. 4. 1409 (1965).

\section{SINGLE ION SOLVENT ACTIVITY COEFFICIENTS}

In the previous tables, I have used single ion solvent activity coefficients to illustrate the various types of solute-solvent interaction. As we all know, it is impossible to measure single ion solvent activity coefficients without making assumptions. However, our understanding of solvent effects on rates and equilibria is no less, whether the assumption we choose is valid or not. What we must do is to use the one assumption consistently in all situations, and secondly, we must record that assumption as part of the discussion. When choosing an assumption, it is desirable that measurements can be made in a large variety of solvents and that the assumption have some intellectually satisfying features. For example, I would not accept an assumption which proposed that the chemical potential of cuprous ion was not changed on transfer through a series of solvents, nor one which claimed that a very large solute was similarly solvated by water and methanol.

The reason that it does not matter whether the assumption is valid or not, is that we are never faced with an equilibrium situation where we have only a single ionic species in solution, so we do not need single ion solvent activity coefficients. We only need the product of activity coefficients for two oppositely charged ions, or the ratio of activity coefficients, for two ions of the same charge. These values are capable of measurement. As we will see, our assumption cancels, provided that it is a constant.

The search for an acceptable assumption is a worthwhile one however, because single ion solvent activity coefficients provide the most convenient way of discussing solvent effects on rates and equilibria. It is an interesting 


\section{A. J. PARKER}

academic exercise also to compare the results of applying different extrathermodynamic assumptions. I think the time has come for us all to make a choice. I hope that this Symposium will give a lead by initiating a procedure which will lead to recommendation of an acceptable assumption. As I see it, the choice should be between one of three assumptions about large inert solutes. I show in parentheses some possible solutes which meet these requirements.

$$
\begin{aligned}
& \text { Large } \\
& \mathrm{o}_{\gamma_{\mathrm{c}}} \mathrm{s}={ }^{\mathrm{o}_{\gamma}} \mathrm{C}_{\mathrm{C}^{+}} \quad \text { Molecule }=\text { cation (Ferrocene) } \\
& { }^{o} \gamma_{\mathbf{R}_{4} \mathrm{C}^{+}}^{\mathrm{s}}={ }^{o} \gamma_{\mathbf{R}_{4} \mathrm{~A}^{-}}^{\mathrm{s}} \quad \text { Cation }=\text { anion }\left(\mathbf{R}_{\mathbf{4}} \mathrm{N}^{+}=\mathbf{R}_{\mathbf{4}} \mathbf{B}^{-}\right) \\
& \mathrm{o}_{\gamma_{\mathrm{A}}^{\mathrm{s}}}^{\mathrm{s}}={ }^{\mathrm{o}_{\gamma_{\mathrm{A}}^{-}}^{\mathrm{s}}} \quad \text { Molecule }=\text { anion }\left(\mathrm{I}_{2}=\mathrm{I}_{3}^{-}\right)
\end{aligned}
$$

The members of each pair should be as similar as possible in all respects, other than in their charge. Strehlow suggested a likely system for the first assumption about molecules and cations, by using the oxidation of ferrocene to ferricinium cation. This is an electroactive system in a variety of solvents and can be studied very conveniently by electrochemical methods. Another possibility is equating the solvent activity coefficients of tetraphenyl methane, or of tetraphenyl tin, with the solvent activity coefficients of tetraphenylarsonium cation.

The second assumption about cations and anions, commonly equates the solvent activity coefficients of tetraphenylarsonium cation with tetraphenyl boron anion. For various reasons, I now prefer equating tetraalkylammonium cations with tetraalkylboron anions. The third assumption about molecules and anions commonly equates the solvent activity coefficients of iodine and triodide ion, or of tetraphenyl methane and tetraphenylboride anion, or of transition state anions and reactants. The three assumptions do not give exactly the same result; but the agreement is tolerable, and qualitative conclusions are not changed drastically, no matter which assumption we choose. The assumptions are compared in Figure 2, which covers changes of more than $10^{16}$ in the solvent activity coefficient of silver cation, on transfer from trifluoroethanol to hexamethylphosphoramide. Here surely is a case for 'chemical interactions' because HMPT and trifluoroethanol have similar dielectric constants. The large anion $=$ large molecule assumption was used to fix the line of arbitrary slope. There are deviations in the solvent activity coefficients of silver cation if we adopt either the large anion-large cation assumption or the large cation-large molecule assumption, but they are not intolerable on the scale considered here, with the exception of the ferrocene assumption in methanol, and the large cation-large anion assumption in HMPT.

It is most important to note another agreeable assumption, which is quite unrelated to any of the assumptions about large solutes. This assumption is that there is no liquid junction potential between half cells in protic solvents, nor is there one between half cells in dipolar aprotic solvents, provided that a suitable bridge is used. This assumption is shown as the filled circles in Figure 2 and only sulpholane leads to the intolerable disagreement with the other three quite unrelated assumptions. All four 


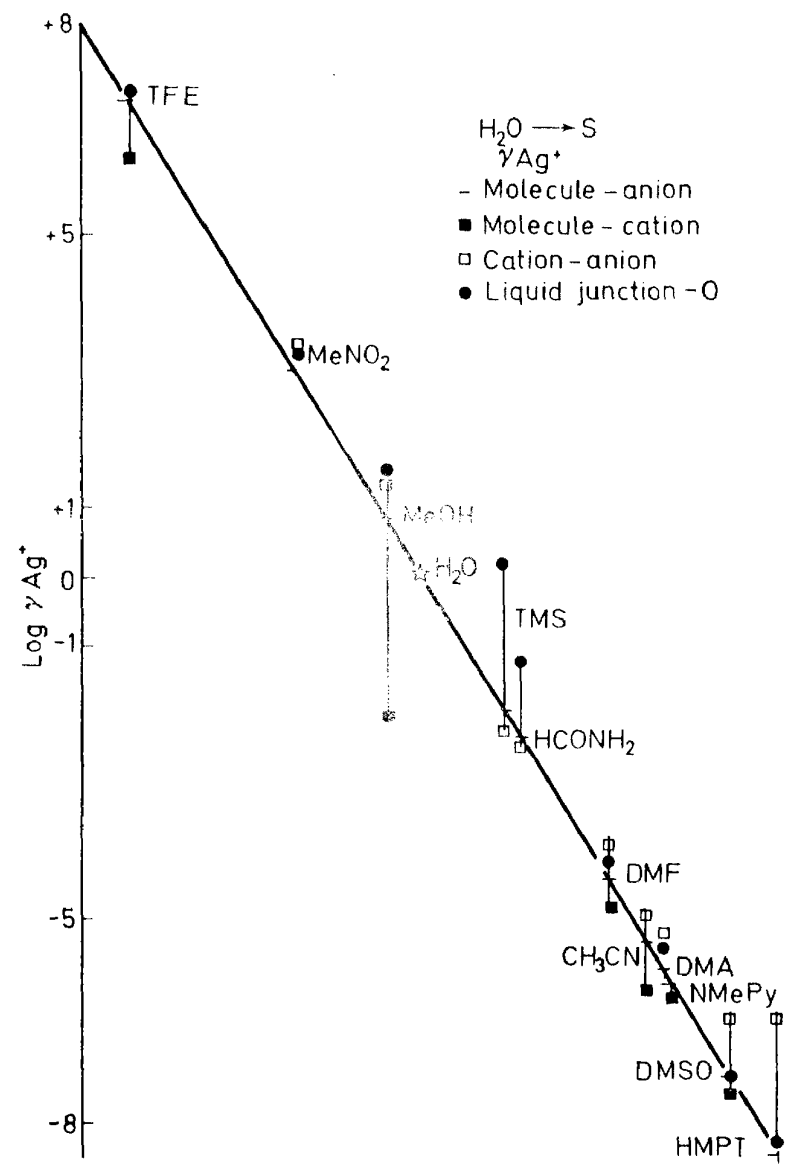

Figure 2. Solvent activity coefficients of silver cation on transfer from water to other solvents at $25^{\circ}$. A comparison of four extrathermodynamic assumptions

assumptions are acceptable on the scale of silver cation solvation, but for more subtle variations of solvation, we need to be more selective.

Trifluoroethanol is an interesting solvent, because it is a very strong hydrogen bond donor and is of low basicity. Some solvent activity coefficients, for transfer of ions from trifluoroethanol to ethanol and dimethylsulphoxide, are in Table 5.

Trifluoroethanol has much the same dielectric constant as ethanol, but differs from ethanol in that it contains a strongly electron-withdrawing $\mathrm{CF}_{3}$ group. For this reason, it is a much stronger hydrogen bond donor than ethanol and a very much stronger hydrogen bond donor than dimethylsulphoxide. Thus it solvates chloride ion more strongly than ethanol and very much more strongly than dimethylsulphoxide. The perchlorate anion is not a hydrogen bond acceptor so that it is similarly solvated by ethanol 


\section{A. J. PARKER}

Table 5. Solvation of ions on transfer from trifluoroethanol at $25^{\circ}$

$$
\underset{\log { }^{\mathrm{TFE}} \gamma^{\mathrm{s}}}{\sin } \gamma_{1_{2}}=\gamma_{1^{-}}
$$

\begin{tabular}{lccc}
\hline Ion & $\mathrm{CF}_{3} \mathrm{CH}_{2} \leftarrow \mathrm{O}^{-} \leftarrow \stackrel{\partial+}{\mathrm{O}}$ & $\mathrm{CH}_{3} \mathrm{CH}_{2}-\stackrel{\partial-}{-\mathrm{O}^{\mathrm{a}}-\mathrm{H}^{\mathrm{b}}}$ & $\left(\mathrm{CH}_{3}\right)_{2}-\stackrel{\partial+}{\mathrm{S}}=\mathrm{O}^{\mathrm{b}}$ \\
\hline $\mathrm{Cl}^{-}$ & 0 & +2.7 & +8.0 \\
$\mathrm{ClO}_{4}^{-}$ & 0 & +0.4 & -2.1 \\
$\mathrm{Ag}^{+}$ & 0 & -5.8 & -13.9 \\
$\mathrm{~K}^{+}$ & 0 & -1.0 & -7.0 \\
\hline
\end{tabular}

a E. Waghorne and A. J. Parker, unpublished.

b. Reference 3 .

and trifluoroethanol and being polarizable, it is more solvated by DMSO than by trifluoroethanol.

The strongly electron-withdrawing $\mathrm{CF}_{3}$ group reduces the basicity of the oxygen atom in trifluoroethanol, so that it is a weaker base than ethanol and is very much weaker than dimethylsulphoxide. Thus silver cation is very much more solvated by ethanol and dimethylsulphoxide than by trifluoroethanol, and the potassium cation shows related, but less pronounced, solvation effects.

Now that we have some understanding of solute-solvent interactions, let us analyse some solvent effects on equilibria in terms of the solvent activity coefficients of all the relevant solutes. A striking example of solvent effects on equilibria is the enormous increase in the stability of mercuric tetrahalide ions on transfer from water to $\mathrm{N}$-methylpyrrolidone, as shown by Buisson and Bréant ${ }^{10}$.

Table 6. Stability constants of mercuric tetrahalides ${ }^{\mathrm{a}}$

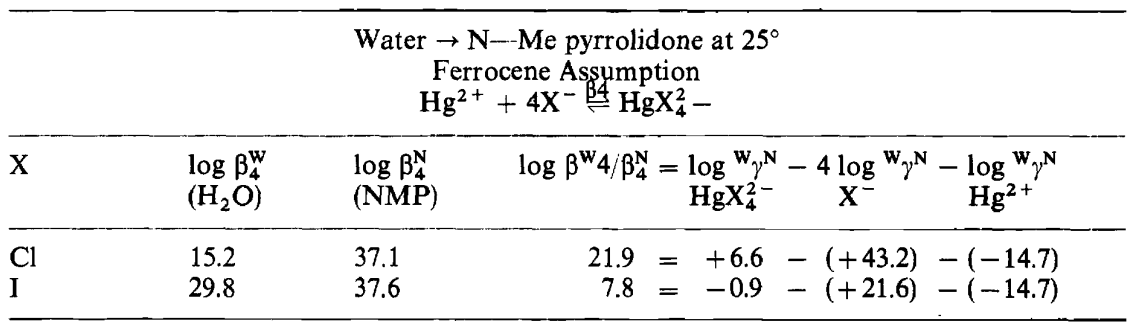

a Reference 10 .

In the first column we notice that aqueous iodide ion forms a much more stable aqueous mercuric tetrahalide ion than does aqueous chloride ion. However, the tetrachloro and tetraiodo complexes are of virtually the same stability in $\mathrm{N}$-methylpyrrolidone as solvent. Obviously, any discussion 
of the relative basicity of halide ions towards mercuric cation must be in terms of the properties of solvated halide ions and solvated complex anions. There is no universal scale of basicity for a set of halide ions, the solvent is of vital importance.

In the third column, we see that the stability of the chloro complex is influenced much more by this solvent transfer than is the stability of the iodo complex. In the remaining columns we see how all the species involved in the equilibria are influenced in different ways by solvent transfer. The mercuric tetrachloride anion is less solvated by $\mathrm{N}$-methylpyrrolidone than by water, $(\log \gamma$ is positive) but the mercuric cation is much more solvated by $\mathrm{N}$-methylpyrrolidone than by water. Both these factors would tend to make the mercuric tetrachloride anion less stable in $\mathrm{N}$-methylpyrrolidone but swamping these, and accounting entirely for the increased stability of the complex in N-methylpyrrolidone, is the decreased solvation (i.e. increased chemical potential) of chloride ion, on transfer from water to $\mathrm{N}$-methylpyrrolidone. The chemical potential of chloride ion is increased more than that of iodide ion on tiansfer, because chloride is a much stronger hydrogen bond acceptor. The complex anions too, show very different behaviour on solvent transfer, according to whether they are iodides or chlorides.

The solvent activity coefficients in Table 6 are from the ferrocene assumption, they are in accord with our discussion of solute-solvent interactions. We note the importance of hydrogen bonding to solvation of halide ions by water, the importance of solvent structure and dispersion forces to solvation of the mercuric tetrahalide ions, and the importance of solvent basicity and softness to the solvation of mercuric cation by $\mathrm{N}$ methylpyrrolidone.

The behaviour of the mercuric halides on solvent transfer is typical of many halide complexes, they are very much more stable in dipolar aprotic than in protic solvents. In view of the substantial changes in equilibrium constants with solvent transfer, it is necessary to question the relevance of Pearson's 'Principle of Hard and Soft Acids and Bases'5. The principle states that 'hard acids prefer to bind to hard bases and soft acids prefer to bind to soft bases.' There is a tendency by some people to take this principle too far. In Table 7 are shown three invalid and three valid tests of the principle.

The first three equilibria show that in water the soft bases iodide and thiocyanate ion bond more strongly to the soft acids iodine, mercury II, and gold I than does the hard chloride ion. However, the basicities of chloride, iodide and thiocyanate towards these soft acids are levelled, and even reversed, on transfer to a variety of dipolar aprotic solvents labelled as $\mathbf{S}$ in the table. Faced with these constants in the last column, we cannot say that soft thiocyanate ion always bonds more strongly than hard chloride ion to the soft acid, gold I. However, Pearson's principle does not require that soft bases should be stronger than hard bases towards soft acids. The principle of hard and soft acids and bases is concerned with the preference of two acids for two bases. The final three equilibria set up the appropriate competitions and are valid tests of the principle. We see, in the first example, that in a competitive situation, there is an enormous preference for hard chloride ion to bond to a hard proton and for soft iodide ion to bond to a soft mercuric cation, no matter what the solvent i.e. equilibrium is well to 


\section{A. J. PARKER}

Table 7. Basicity of soft $\mathrm{I}^{-}$and $\mathrm{SCN}^{-}$and of hard $\mathrm{Cl}^{-}$towards soft acids

Invalid Tests

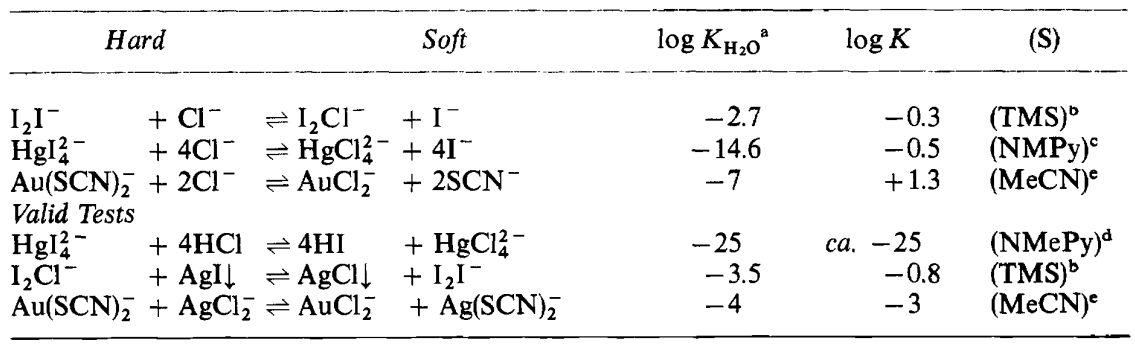

atability Constants The Chemical Society, London, Special Publication No. 17 (1964).

b M. Deneux and R. L. Benoit. ICCC Abstracts Conference Sydney 1969, p. 104; ibid. Can. J. Chem. (1970).

c Ref. 10.

${ }^{d}$ Estimated from expected solvent activity coefficients of $\mathrm{Hl}$ and $\mathrm{HCl}$ and from data in reference 10

- A. D. Goolsby and D. T. Sawyer, Anal. Chem. 40, 1978 (1968); O. Bravo and R. T. Iwamoto. Inorg. Chim. Acta. 3, 663 (1969).

the left. In the second example, silver cation is a softer acid than iodine because it prefers to bond to soft iodide ion, but this tendency is levelled significantly by transfer from 'hard' water to 'soft' sulpholane. In the final example, gold I is softer than silver I, because it prefers to bond to the softer thiocyanate ion, leaving the harder chloride ion with silver, but this tendency is slightly greater in 'hard' water than in soft acetonitrile.

Although I have many objections to the Principle of Hard and Soft Acids and Bases, it must be acknowledged that the very large effects of solvent on equilibria do not seem to require major changes in the original classification of acids and bases as hard or soft. This despite the fact that nearly all the original classifications were made from data measured in aqueous solution.

\section{REDOX SYSTEMS}

As you might expect, redox equilibria are strongly influenced by solvent transfer. Thus it has been known for a long time that cupric nitrate or cupric perchlorate in acetonitrile will oxidise and dissolve metallic silver, despite the fact that this is energetically unfavourable by 0.64 volt in water.

Table 8. The silver-copper redox equilibrium

\begin{tabular}{|c|c|c|}
\hline \multicolumn{3}{|c|}{$\begin{array}{c}\text { Water } \rightarrow \text { Acetronitrile at } 25^{\circ} \text { a } \\
\text { Ferrocene Assumption } \\
\mathrm{Ag}^{+}+\mathrm{Cu}^{+} \rightleftharpoons \mathrm{Ag}^{0}+\mathrm{Cu}^{2+}\end{array}$} \\
\hline $\begin{array}{c}\Delta \mathrm{E}^{\circ}(\mathrm{MeCN})_{\text {volt }} \\
\quad+0.41\end{array}$ & $\begin{aligned}-\Delta \mathrm{E}^{\circ}\left(\mathrm{H}_{2} \mathrm{O}\right) & =0.059\left(\log \mathbf{w}_{\gamma^{\mathrm{A}}} \mathrm{cu}^{2+}-\log \mathbf{w}_{\gamma^{\mathrm{A}}} \mathrm{cu}^{+}+(-12.5)\right. \\
-(-0.64) & =0.059[+1.0-(.0)\end{aligned}$ & $\begin{array}{l}\left.-\log \mathbf{w}_{\gamma^{\mathrm{A}}}{ }_{\mathrm{Ag}}^{+}\right) \\
-(-5.50)]\end{array}$ \\
\hline
\end{tabular}

As shown in Table 8, the redox equilibrium between copper I and silver I is changed by more than 1 volt $\left(10^{18}\right.$ in the equilibrium constant) on solvent transfer. This is due almost entirely to the much greater solvation of silver I and copper I cations in acetronitrile than in water, as shown by their very 
small solvent activity coefficients (negative $\log \gamma$ ). The closed shell copper II cation is slightly less solvated by acetonitrile than by water, and this also contributes in the same direction to the change in equilibrium constant.

It is hardly necessary to stress the practical applications of carrying out a redox reaction, such as this, towards the left in a water miscible dipolar aprotic solvent, then reversing the process to the right by diluting with water, then recovering the original oxidant (in this case silver metal) and finally recovering the original reductant in the dipolar aprotic solvent, by distillation.

\section{ASSOCIATIVE EQUILIBRIA}

I want now to show how we can use solvent activity coefficients to obtain information about the nature of various associated species in solution. One example, which provides us with all the species that we are interested in, is transfer of the associative $\mathrm{S}_{\mathrm{N}} 2$ reaction between an anion and a cation, from the protic solvent ethanol to the dipolar aprotic solvent, dimethylacetamide. In the solutions we have separated ions, ion pairs, a transition

Table 9. Solvation of species in an $\mathrm{S}_{\mathrm{N}} 2$ reaction ${ }^{\mathrm{a}}$ Ethanol to dimethylacetamide at $25^{\circ}$

\begin{tabular}{|c|c|c|c|}
\hline \multicolumn{4}{|c|}{ No Assumptions } \\
\hline $\begin{array}{l}\text { Species: } \\
\log \mathrm{E}^{\mathrm{E}} \mathbf{D}^{\mathrm{D}}\end{array}$ & $\begin{array}{cc}\left(\mathrm{Me}_{3} \mathrm{~S}^{+}+\mathrm{Br}^{-}\right) & \mathrm{Me}_{3}^{+} \mathrm{S} \cdot \mathrm{Br} \\
+0.9 & +0.9 \\
\text { ions } & \text { ion pair }\end{array}$ & $\begin{array}{c}\stackrel{\delta-}{\mathrm{Br}-}-\mathrm{Me}--\mathrm{S} \\
-1.4 \\
\quad \text { transition state }\end{array}$ & $\begin{array}{c}\delta+\neq \\
\mathrm{SMe}_{2} \rightleftharpoons \mathrm{MeBr}+\mathrm{Me}_{2} \mathrm{~S} \\
-0.5 \\
\text { products }\end{array}$ \\
\hline
\end{tabular}

a Reference 3.

state, and products. From a mechanistic viewpoint, it is of interest to know if the transition state is like the reactants, either like solvent-separated ions or like an ion pair, or if it is like the products as a polar molecule. A third possibility is that the transition state is like some other species, quite different from either reactants or products. Intuitively one might favour the ion pair as a model, because the two ions must come together to react.

In Table 9 we show the solvent activity coefficients for the transition state and other species. It is clear that the $S_{N} 2$ transition state does not respond to solvent transfer like an ion pair, nor like solvent separated ions. The solvent activity coefficient of -1.4 for the transition state is the most negative of all the species, but it is nearest to that of the products. Values like this are obtained for transfer of very polar solutes from ethanol to dimethylacetamide, e.g. 4-iodonitrobenzene. It would seem that the best model for this $\mathrm{S}_{\mathrm{N}} 2$ transtion state is a large polarizable uncharged solute. We can make the important observation that there seems to be very little negative charge localised on bromine in this transition state. If the transition state contained a solvated partial bromide ion, then we would expect a more positive solvent activity coefficient for transfer from a protic to a dipolar aprotic solvent.

You will have noticed that the trimethylsulphonium bromide ion pair responds to solvent transfer, from ethanol to dimethylacetamide, just as if it 


\section{A. J. PARKER}

were a separated anion and cation. This is I think a most important distinction between ion pairs formed by alkyl substituted cations like ammonium, sulphonium, and carbonium, and ion pairs formed by alkali metal cations like lithium and sodium. The ion pairs formed by alkyl substituted cations are very loose, i.e. they behave as though they were a solvent separated anion and cation, whereas the ion pairs formed by alkali metal cations are 'tight', they could be described as intimate ion pairs. The recognition of at least two types of ion pair, according to the nature of the cation, is of interest to a physical organic chemist because carbonium ions may be like alkyl substituted cations. This may be relevant to the work of the late Saul Winstein, who proposed intimate ion pairs and solvent separated ion pairs in the solvolysis reactions of organic compounds.

Certainly the ion pairs formed between tetraalkylammonium cations and halide ions are very different from the ion pairs formed between lithium cations and halide ions. Some compelling evidence is shown in Figure 3.
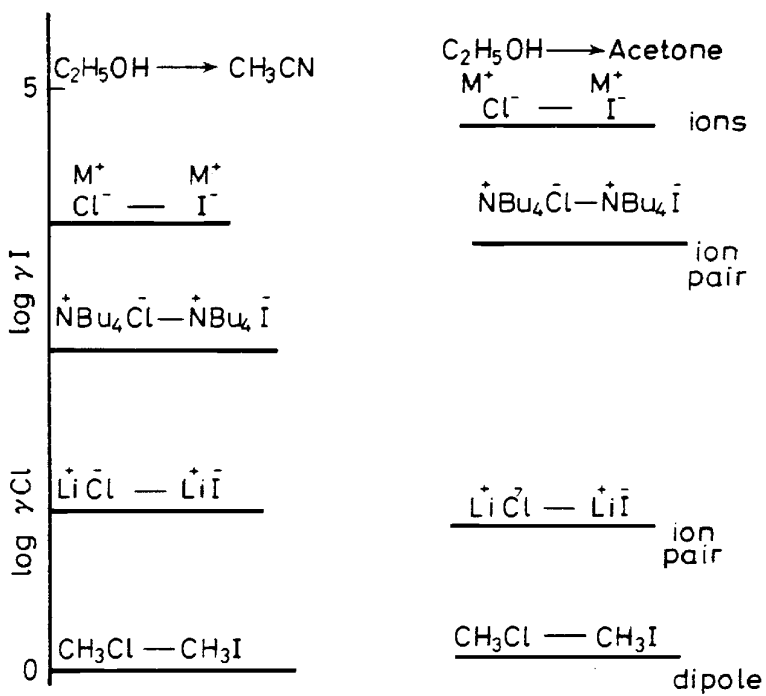

Figure 3. Solvent activity coefficients of chlorine and iodine containing species on transfer from ethanol to a dipolar aprotic solvent

We show the difference between the solvent activity coefficients, within four pairs of chlorine containing species and iodine containing species, on transfer from ethanol to a dipolar aprotic solvent. The four pairs are a chloride and an iodide ion with a common dissociated cation, $\mathbf{M}^{+}$, a tetrabutylammonium chloride and iodide ion pair, a lithium chloride and lithium iodide ion-pair, and the methyl chloride and methyl iodide dipolar molecules. So as to obtain information about these solutes, we apply the principle that the greater the localization of negative charge on the halogens in the particular pair of solutes, then the greater will be the difference between the solvent activity coefficients of the chlorine and iodine containing species. 
This principle follows because, as shown, in the extreme case where negative charge is localized on chlorine in the chloride ion and on iodine in the iodide ion, the solvent activity coefficients of two electrolytes differ by between $10^{4}$ and $10^{5}$. At the other extreme, in the methyl halides, in which very little negative charge is localized on the halogen, we see that the solvent activity coefficients for methyl chloride and methyl iodide differ by less than a factor of two. Now let us look at the ion pairs, how localised is negative charge on the halogens? The two types of ion pair behave differently. The lithium halide ion pairs tend to respond to solvent transfer like the methyl halides, i.e. like very polar covalent molecules. The tetraalkylammonium halide ion pairs however, respond to solvent transfer more like dissociated ions than like dipolar molecules. This behaviour seems to be quite general for a variety of alkyl substituted cations versus alkali metal cations in a variety of protic and dipolar aprotic solvents. It is not an effect peculiar to lithium halide ion pairs. The same principle can be applied to species like silver chloride and silver iodide in solution, do they have negative charge localized on halogen, or do they behave like covalent molecules? As noted, the best explanation for the behaviour shown in Figure 3 is that lithium halide ion pairs are 'tight' and tetraalkylammonium ion pairs are 'loose'.

It follows that, since one must consider different solvent activity coefficients for ion pairs, one must consider also the conventional activity coefficients of ion pairs, as well as activity coefficients of dissociated ions, when calculating ion pair association constants, or degrees of dissociation, at different ionic strengths in the one solvent. In many treatments, the activity coefficient of an ion pair is assumed to be unity.

\section{ENTHALPY AND ENTROPY OF TRANSFER ${ }^{11,12}$}

Up until now, we have dealt with changes in the standard chemical potentials of solutes when they are transferred from one solvent to another at $25^{\circ}$. Now we will look at enthalpies and entropies of transfer from water to dimethylsulphoxide and see how they combine to give free energy of transfer. Some data are given in Table 10. Again we see very different behaviour on the part of alkali metal salts, versus a tetraalkylammonium salt of the same anion, on transfer from water to dimethylsulphoxide. Enthalpies of transfer are more positive for tetraalkylammonium chlorides and iodides than for lithium chloride or cesium iodide.

Table 10. Free energics, enthalpies and entropies of transfer

\begin{tabular}{lccc}
\hline \multirow{1}{*}{ Salt } & \multicolumn{2}{c}{ Water $\rightarrow$ DMSO at $25^{\circ}$} & \\
& \multicolumn{1}{c}{$\Delta G_{\mathrm{tr}}$} & $\Delta H_{\mathrm{tr}}^{\mathrm{b}}$ & $\Delta S_{\mathrm{tr}}$ \\
& $\mathrm{Kcal}$. & $\mathrm{Kcal}$. & e.u. \\
\hline $\mathrm{LiCl}$ & $+4.9^{\mathrm{b}}$ & -2.0 & -23 \\
$\mathrm{NBu}_{4} \mathrm{Cl}$ & $+1.7^{\mathrm{a}}$ & +11.0 & +31 \\
$\mathrm{CsI}$ & $-0.4^{\mathrm{a}}$ & -10.8 & -35 \\
$\mathrm{NEt}_{4} \mathrm{I}$ & $-5^{\mathrm{a}}$ & -2 & +10 \\
\hline
\end{tabular}

a Reference 3

b Reference 12 .

c J. N. Butler and J. C. Synnott. J. Amer. Chem. Soc. 92. 2602 (1970). 


\section{A. J. PARKER}

There is a substantial decrease in the partial molar entropy when both lithium chloride and cesium iodide, are transferred from water to dimethylsulphoxide. This decrease in entropy is typical of the behaviour of most salts of the alkali metals, on transfer from water to dimethylsulphoxide, and the effect is to increase the solvent activity coefficients of these salts in dimethylsulphoxide, relative to water. It has been claimed that lithium, sodium, potassium, and cesium cations are structure breakers in water, the decrease in entropy when their salts are transferred to dimethylsulphoxide may indicate that they are structure makers in dimethylsulphoxide.

In marked contrast to the alkali metal halides, there is a very substantial increase in the partial molar entropy of tetrabutylammonium chloride and tetraethylammonium iodide, on transfer from water to dimethylsulphoxide. This increase in entropy is typical of the behaviour of most tetraalkylammonium salts on this solvent transfer. The tendency is to decrease the solvent activity coefficients of tetraalkylammonium salts in dimethylsulphoxide, relative to water. It has been claimed that tetraalkylammonium cations are structure makers in water, the increase in entropy when their salts are transferred to dimethylsulphoxide may indicate that perhaps they are structure breakers in dimethylsulphoxide. The positive entropy of transfer explains why tetraalkylammonium salts are conveniently recrystallised from dipolar aprotic solvents, the solubility in dipolar aprotic solvents increases strongly on heating. The differences in entropies of transfer between alkali metal salts and between tetraalkylammonium salts of the same anion are more than 50 entropy units in some cases. This is an enormous difference,

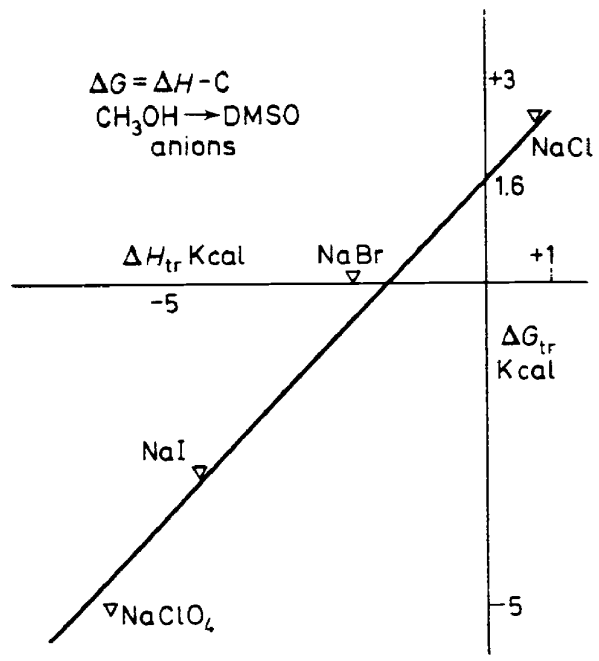

Figure 4. Linear relationship between free energy and enthalpy of transfer of sodium salts from methanol to dimethylsulphoxide. $\Delta G_{\mathrm{tr}}=\Delta H_{\mathrm{tr}}-$ constant

and needs further investigation as to structural interactions between solutes and solvents.

We cannot use just any cation as a model for solvation of another. For 
example, we should use tetraalkyammonium or trialkylsulphonium cations as models for carbonium ions. If we use alkali metal cations misleading conclusions are likely.

Are the effects of protic and dipolar aprotic solvents on the standard chemical potential of anions an enthalpy or an entropy effect, or a combination of both? We can obtain a picture of effects of solvent transfer on solvation of anions by examining a series of salts of the same cation as in Figure 4, where the expression is plotted for transfer of some sodium salts.

$$
\Delta G_{\mathrm{tr}}=\Delta H_{\mathrm{tr}}-T \Delta S_{\mathrm{tr}}=\Delta H_{\mathrm{tr}}-\text { constant }
$$

It is seen that the free energy of transfer is a linear function of the enthalpy of transfer. It also has unit slope. Thus an equation which assumes constant $T \Delta S$ is followed quite well. This link between enthalpy and free energy is more or less typical of many anions in a variety of solvents, the entropies of transfer are virtually the same (TAS is $1.6 \mathrm{Kcals})$ for transfer of a series of anions associated with the same cation, from methanol to dimethylsulphoxide.

The picture is different for cations, however. Neither the entropy of transfer nor the enthalpy of transfer are constant, when free energies of transfer of different salts of iodide ion are compared, as in Figure 5. Here the cation is the variable, and a plot of free energy of transfer versus enthalpy of transfer, for transfer of a series of iodides from methanol to DMSO, is a scatter diagram. The point for tetrabutylammonium iodide is particularly unrelated to the other iodides.

The changes shown in the preceding figures are quite general. The large changes in anion solvation, on transfer from protic to dipolar aprotic solvents, are reflected much more in enthalpies of transfer rather than in

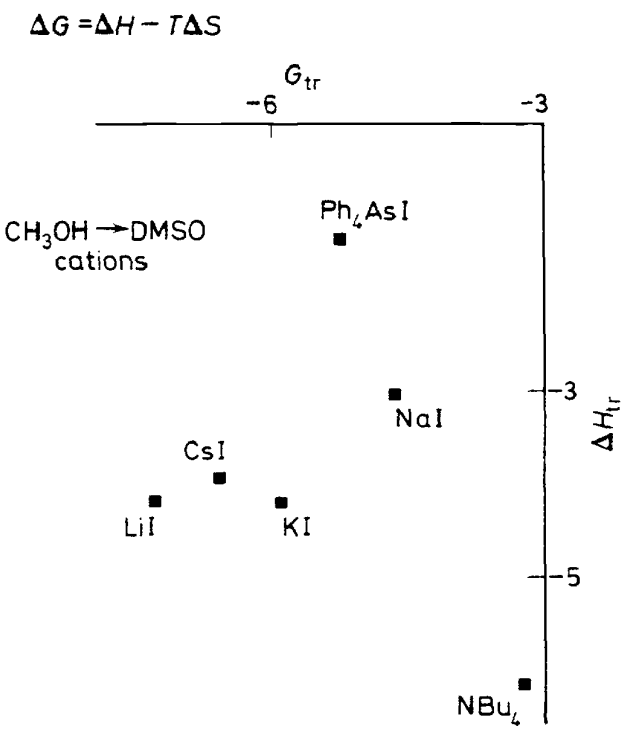

Figure 5. Absence of linear relationship between free energy, entropy, or enthaipy of transfer of iodides from methanol to dimethylsulphoxide. $\Delta G_{\mathrm{tr}}=\Delta H_{\mathrm{tr}}-T \Delta S_{\mathrm{tr}}$ 


\section{A. J. PARKER}

entropies of transfer. The entropies of transfer do show some variation, methanol to dimethylsulphoxide has less variation than the entropies of transfer of anions from methanol to dimethylformamide, for example, but it is a useful generalisation to relate the hydrogen bonding solvation effect to an enthalpy change.

As a final example of the use of solvent activity coefficients to elucidate mechanism, we will compare the solvation of two transition state anions, the chloride anion, and the silver dichloride anion. The transition states are for chloride exchange at a saturated carbon atom and at platinum II and we are transferring the reactions from methanol to dimethylsulphoxide.

The solvent activity coefficients of the four chloride containing species range over $10^{7}$ units. Our interpretation of the figures shown in Table 11, is that the silver dichloride anion has negative charge delocalised over the whole large polarizable anion and so is not a good hydrogen bond acceptor. Thus it is more solvated by DMSO than by methanol. The transition state

Table 11. Solvation of transition states

Displacements at carbon and platinum ${ }^{2}$ $\mathrm{MeOH} \rightarrow$ DMSO at $25^{\circ}$

\begin{tabular}{lccl}
\hline \multicolumn{4}{c}{ Assume $\gamma_{\mathrm{I}_{2}}=\gamma_{\mathrm{t}_{3}}$} \\
\hline $\mathrm{Cl}^{-}+\mathrm{CH}_{3} \mathrm{Cl}$ & $\rightarrow \mathrm{ClCH}_{3} \mathrm{Cl}^{-\neq}$ \\
$\left.\mathrm{Cl}^{-}+t-\mathrm{Pt}^{-} \mathrm{PEt}_{3}\right)_{2} \mathrm{Cl}_{2}$ & $\rightarrow \mathrm{Cl}^{-}--\mathrm{PtCl}_{2}\left(\mathrm{PEt}_{3}\right)_{2}^{\neq} \rightarrow$ Product \\
$\mathrm{Cl}^{-}+\mathrm{AgCl}$ & $\rightarrow \mathrm{ClAgCl}^{-}$ \\
\hline \multicolumn{4}{c}{} \\
$\mathrm{ClAgCl}^{-}$ & $\log \gamma-$ & \\
-1.3 & $\mathrm{ClCH}_{3} \mathrm{Cl}^{-\neq}$ & $\mathrm{ClPtCl}_{2}\left(\mathrm{PEt}_{3}\right)_{2}^{-\neq}$ & $\mathrm{Cl}^{-}$ \\
tight & +1.2 & +3.6 & +5.5 \\
\hline
\end{tabular}

${ }^{a}$ A. J. Parker, unpublished work, cf. reference 3.

anion for exchange at carbon has a little negative charge localized on chlorine, so it is slightly more solvated by methanol than by DMSO. The transition state anion for exchange at platinium II has considerable negative charge localized on the entering chloride ion, so like chloride ion, it is much more solvated by methanol than by dimethylsulphoxide. It follows that the $\mathrm{C}-\mathrm{Cl}$ bond is much tighter than the $\mathrm{Pt}-\mathrm{Cl}$ bond in the respective transition states.

\section{References}

1 J. Miller and A. J. Parker. J. Amer. Chem. Soc. 83, 117 (1961).

2 A. J. Parker. Quart. Rev. London 16, 163 (1962).

3 A. J. Parker. Chem. Rev. 69, 1 (1969).

4 R. Alexander, Thesis, University of W. A., Nedlands, Western Australia, 1968.

5 R. G. Pearson. Survey of Progress in Chemistry 5, 1 (1969).

6 M. Alfenaar and C. L. de Ligny. Recueil 86, 929 (1967).

7 C. Barraqué, J. Vedel and B. Trémillon. Bull. Soc. Chim. France, 3421 (1968). 
IONIC EQUILIBRIA IN PROTIC AND IN DIPOLAR APROTIC SOLVENTS

8 J. F. Coetzee and J. J. Campion. J. Amer. Chem. Soc. 89, 2513 (1967);

J. F. Coetzee, J. M. Simon and R. J. Bertozzi. Analyt. Chem. 41, 766 (1969).

9 V. A. Pleskov. Usp. Khim 16, 254 (1947).

10 M. Bréant, C. Buisson, M. Porteix, J. L. Sue and J. P. Terrat, J. Electroanal. Chem. 24, 409 (1970).

${ }^{11}$ G. Choux and R. L. Benoit. J. Amer. Chem. Soc. 91, 6221 (1969).

12 R. Fuchs, J. L. Bear and R. F. Rodewald. J. Amer. Chem. Soc. 91,5797 (1969). 